\title{
Modified hypoosmotic swelling test for the assessment of boar and bull sperm sensitivity to cryopreservation
}

\author{
Petra Přinosilová, Věra Kopecká, Jaroslava Hlavicová, Monika Kunetková \\ Veterinary Research Institute, Brno, Czech Republic \\ Received April 22, 2014 \\ Accepted September 17, 2014
}

\begin{abstract}
Routine methods for the evaluation of sperm quality are not sufficiently useful to determine the sensitivity of sperm cells to cold shock. The aim of our preliminary study was to determine whether the sperm plasma membrane integrity evaluated by modified hypoosmotic swelling test based on simple hypoosmotic swelling test (HOS test) and eosin-nigrosin staining could be helpful in predicting the degree of boar sperm survivability during semen cryopreservation. Ejaculates collected from 24 boars and 20 bulls were used in the experiment. Fresh ejaculates were evaluated by routine sperm analysis and a modified HOS test, and subsequently frozen. Sperm cryosurvivability was defined as the percentage of motile spermatozoa that survived the freezing process. A higher percentage of sperm was recovered after the thawing of semen with a higher percentage of HOS-positive and eosin-negative sperm $(P<0.01)$. Both indicators were found to be correlated $(\mathrm{r}=0.707$ and $\mathrm{r}=0.705$, respectively; $P<0.01)$. Moreover, the percentage of HOS-positive and eosin-negative sperm was similar to the percentage of viable sperm after thawing as determined by traditional eosin-nigrosin staining in boars $(50.90 \pm 9.88 \%$ and $49.31 \pm 11.63 \%$, respectively) and bulls $(55.91 \pm 10.34 \%$ and $55.63 \pm 6.64 \%$, respectively) and both indicators showed a positive correlation $(\mathrm{r}=0.558$ and $\mathrm{r}=0.504$, respectively; $P<0.05)$. In conclusion, based on the obtained results, we can assume that the modified HOS test can detect differences in sperm membrane resistance which allows assessment of semen quality from the perspective of its further use, e.g. cryopreservation.
\end{abstract}

Ejaculate, membrane, viability, survival test, freezing, semen analysis

As the use of artificial insemination with preserved semen forms a large part of reproductive technologies, production of high-quality insemination doses is of major interest. Various approaches to improving the quality of frozen-thawed sperm have been used in relation to changes in the holding times before freezing; new packaging systems; addition of various additives; use of intrauterine artificial insemination etc. However, there is great variability among individual animals in sustaining sperm cryopreservation.

Cryopreservation of semen is associated with different injuries to the spermatozoa, such as cold shock, osmotic stress, cryoprotectant intoxication and intracellular ice crystal formation during freezing and thawing. Therefore, the cryopreservation process results in reduced fertility compared to fresh semen. Boar spermatozoa, especially, are characterized by marked inter-individual differences in their resistance to cold shock and freezing (Holt et al. 2005). In comparison with other farm animals, boar spermatozoa have a higher unsaturated/saturated fatty acid ratio and lower cholesterol content, and thus their response to decreasing temperatures is stronger (Parks and Lynch 1992).

We need to identify those studs and/or ejaculates that will better survive the preservation process. The question that motivated our study was as to whether we were able to predict the ability of sperm to survive the preservation process merely on the basis of the assessment of fresh semen. However, routine methods for the evaluation of semen quality are considered insufficient to determine the sensitivity of sperm cells to cold shock (Amann 1989; Roca et al. 2006; Casas et al. 2010).

Address for correspondence:

MVDr. Petra Přinosilová, Ph.D.

Laboratory for Spermatology and Andrology

Department of Genetics and Reproduction

Veterinary Research Institute, Hudcova 70, 62100 Brno 
Sperm plasma membrane integrity is commonly evaluated by intravital staining tests documenting a change in the permeability of the plasma membrane or by a test of membrane tolerance and resistance to hypo-osmotic conditions (HOS test). Simple HOS test, introduced by Jeyendran et al. (1984) is still widely used for the evaluation of both human and animal semen. The rationale of the test is based on the assumption that after the passage of fluid into an undamaged membrane of a live sperm under hypoosmotic conditions, coiling of sperm tails occurs. However, poor quality spermatozoa with borderline membrane integrity will exhibit swelling in simple HOS test, thereby yielding false positive results. By combining supravital staining with HOS test, based on eosin-nigrosin staining of semen samples that underwent HOS test and eosin-nigrosin staining, the number of false positive results can be reduced (Chan et al. 1991). Moreover, modified HOS test (mHOST) brings more information about the integrity of the whole sperm membrane because HOS test gives information regarding the sperm tail membrane integrity, while the sperm viability test is more specific in evaluating the integrity of the sperm head membranes. It has been shown that the membranes in the head and tail compartments have different lability (Hammerstedt 1979).

Accordingly, based on the above mentioned facts, the aim of our preliminary study was to investigate whether plasma membrane integrity, evaluated by mHOST, can be helpful in predicting the degree of boar and bull sperm survivability during semen cryopreservation.

\section{Materials and Methods}

Fresh bull (Experiment 1) and boar (Experiment 2) ejaculates were evaluated by routine sperm analysis and by modified HOS test and were then frozen in straws.

The standard fresh semen analysis included total sperm count, total sperm motility and progressive sperm motility, and plasma membrane integrity (viability) - evaluated by eosin nigrosin staining (World Health Organization 2010). Semen volume was measured using a graduated vial and concentration was measured in a Bürker chamber. Sperm motility (and progressive motility) was analysed under an optical microscope at $\times$ 200 magnification. Sperm morphology was evaluated according to Tygerberg's strict criteria (Kruger et al. 1986). Samples were stained for sperm morphology analysis according to Farelly (smears were fixed in $3.5 \%$ formalin and stained with $5 \%$ aniline blue for $10 \mathrm{~s}$ and $0.5 \%$ crystal violet for $6 \mathrm{~s}$ ) and evaluated with the use of the SASMO computer program (Strict Analysis of Sperm Morphology; Veznik et al. 2001). The analysis of sperm motility and viability was carried out also after the semen samples were thawed.

The principle of modified HOS test is based upon the addition of $0.1 \mathrm{ml}$ of fresh semen to $1 \mathrm{ml}$ of a hypoosmotic solution equilibrated to $37^{\circ} \mathrm{C}$ (World Health Organization 2010). The sample was then incubated at $37{ }^{\circ} \mathrm{C}$ for $30 \mathrm{~min}$. After incubation and mixing, the sample was smeared on a slide. Additional staining with $0.5 \%$ eosin and $10 \%$ nigrosin was carried out before microscopic evaluation at $\times 1000$ magnification, using oil immersion. At least 200 spermatozoa were evaluated.

Having performed modified HOS test, we obtained the following main three sperm categories: 1) HOS-positive and eosin-negative, i.e. live spermatozoa with good membrane resistance, 2) HOS-negative and eosin-positive, i.e. dead spermatozoa and 3) HOS-positive and eosin-positive, i.e. viable spermatozoa but with reduced plasma membrane resistance (Plate V, Fig. 1).

\section{Experiment 1: Determination of boar sperm sensitivity to cryopreservation}

The ejaculates of 24 boars (Pietren and Large White breed of proven fertility) were collected by the glovedhand technique. An aliquot of each semen sample was gently mixed with BTS medium (Beltsville thawing solution, Minitube, Tiefenbach, Germany) at a $1: 1$ ratio (v/v) and transported in an insulated container at $17{ }^{\circ} \mathrm{C}$ within $1 \mathrm{~h}$ to the laboratory and immediately assessed. Only ejaculates with sperm motility higher than $60 \%$ were used in the experiment. Ejaculates were frozen using the standard procedure as described by Westendorf et al. (1975) and modified by Thurston et al. (1999). Briefly, after $20 \mathrm{~h}$ storage at $17^{\circ} \mathrm{C}$ the extended semen was centrifuged at $800 \times g$ for $10 \mathrm{~min}$. The supernatant was discarded and the pellet was resuspended in a cooling extender $(0.24 \mathrm{M}$ lactose, $20 \%$ egg yolk (v/v) and $100 \mu \mathrm{g} / \mathrm{ml}$ kanamycin sulfate) at a ratio of $3 / 5$ of the final volume and cooled to $5{ }^{\circ} \mathrm{C}$ within $2 \mathrm{~h}$. Subsequently, the freezing extender ( $0.24 \mathrm{M}$ lactose, $20 \%$ egg yolk (v/v), $7.5 \%$ glycerol (v/v), $1.3 \%$ Equex STM (v/v), Minitube, Tiefenbach, Germany, and $100 \mu \mathrm{g} / \mathrm{ml}$ kanamycin sulfate) was added at a ratio of $2 / 5$ of the final volume, thus giving final glycerol concentration of $3 \%$. The final sperm concentration was $0.5 \times 10^{9}$ spermatozoa/ $\mathrm{ml}$. The semen was loaded into $0.5 \mathrm{ml}$ straws (Minitube, Tiefenbach, Germany), sealed and placed in liquid nitrogen vapour at $4 \mathrm{~cm}$ above the nitrogen level for $20 \mathrm{~min}$, then plunged into liquid nitrogen and stored until use. After at least 2-4 weeks of storage, samples were thawed in a water bath at $38^{\circ} \mathrm{C}$ for $30 \mathrm{~s}$ and emptied into BTS medium to reach the final sperm concentration of $100 \times 10^{6}$ spermatozoa $/ \mathrm{ml}$. 
Sperm cryosurvivability was defined as the percentage of motile spermatozoa that survived the freezing process $(\%$ of frozen/thawed sperm motility $/ \%$ of fresh sperm motility $\times 100)$.

Group with higher survival rate (Good freezers): $\geq 50 \%$; group with lower survival rate (Bad freezers): $<50 \%$

Experiment 2: Determination of bull sperm sensitivity to cryopreservation

The ejaculates of 20 Holstein bulls of proven fertility housed in one insemination centre were used in the experiment. Only fresh ejaculates with initial fresh sperm motility higher than $70 \%$ were assigned for the cryopreservation. The semen analysis including mHOST was performed immediately after semen collection. Semen was cryopreserved according to the standard procedure applied at the insemination centre using Optidyl ${ }^{\circledR}$, a commercial egg yolk freezing extender (Cryo-Vet, Quebriac, France), packed in $0.25 \mathrm{ml}$ straws and frozen in a programmable freezer.

After at least 2-4 weeks of storage, the straws were thawed in a water bath at $38{ }^{\circ} \mathrm{C}$ for $30 \mathrm{~s}$ and emptied into saline to reach the final sperm concentration of $100 \times 10^{6} \mathrm{spermatozoa} / \mathrm{ml}$.

Sperm cryosurvivability was defined as the percentage of motile spermatozoa that survived the freezing process $(\%$ of frozen/thawed sperm motility $/ \%$ of fresh sperm motility $\times 100)$.

Group with higher survival rate (good freezers): $\geq 70 \%$; group with lower survival rate (bad freezers): $<70 \%$.

All statistical analyses were performed with SPSS software (Version 18.0 for Windows, SPSS Inc., Chicago, IL, USA). If the data were normally distributed according to Kolmogorov-Smirnov test, Student's $t$-test and paired $t$-test were used for comparison between groups. Nonparametric Mann-Whitney U test and Wilcoxon signed rank test were used in the case of violation of normal distribution. Spearman's correlation was used to assess the relationship between sperm indices. The $P$-values of $<0.05$ and $<0.01$ were considered statistically significant.

\section{Results}

Experiment 1: Determination of boar sperm sensitivity to cryopreservation

Results of semen analysis for fresh and frozen boar semen varying in cryosurvivability are shown in Table 1 . The recovery rate after thawing was higher in the semen samples which had a higher percentage of HOS-positive and eosin-negative sperm $(P<0.01$, Fig. 2) and both indices showed a positive correlation $(\mathrm{r}=0.707, P<0.01)$. Other indices of fresh sperm analysis did not differ significantly.

The percentage of HOS-positive and eosin-negative spermatozoa $(50.90 \pm 9.88 \%)$ when evaluating ejaculates all together was similar to that of viable sperm after thawing, as determined by eosin-nigrosin staining $(49.31 \pm 11.63 \% ; P=0.410)$ and both indices were in correlation $(\mathrm{r}=0.558, P<0.05)$.

Experiment 2: Determination of bull sperm sensitivity to cryopreservation

Results of semen analysis for fresh and frozen bull semen varying in cryosurvivability are shown in Table 2.

Evaluating the relationship between the percentage of the HOS-positive and eosin-negative sperm category and the resistance of bull sperm to freezing, we obtained a significant relationship $(\mathrm{r}=0.705, P<0.01)$. The 


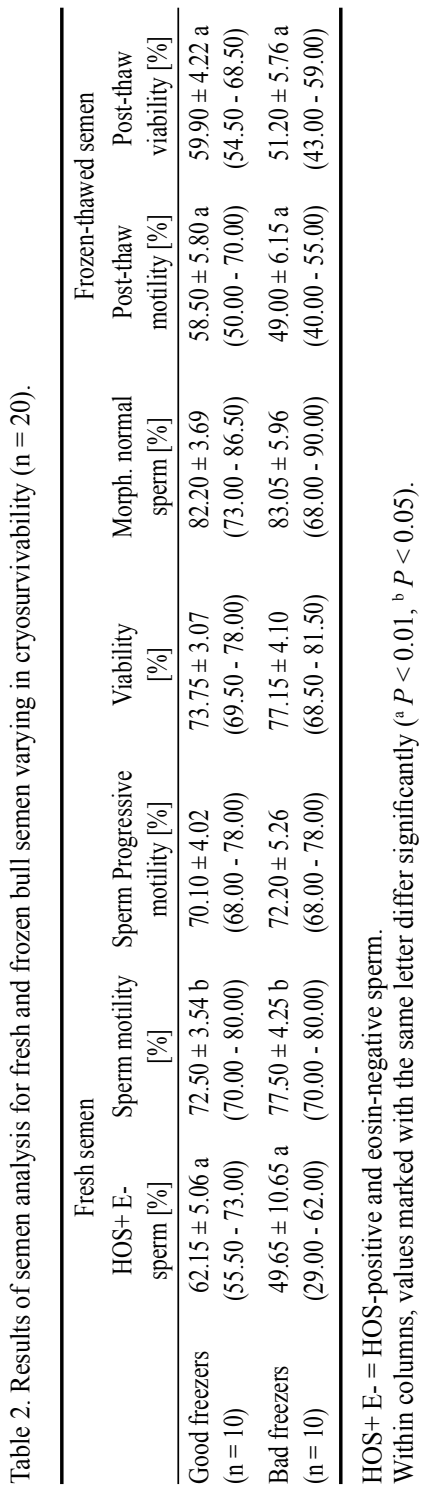

recovery rate after thawing was higher in bull ejaculates with a higher percentage of HOS-positive and eosinnegative sperm $(P<0.01$, Fig. 3$)$. Other indices of fresh sperm analysis did not differ significantly.

The percentage of HOS-positive and eosin-negative spermatozoa $(55.91 \pm 10.34 \%)$ when evaluating ejaculates all together was similar to that of viable sperm after thawing as determined by eosin-nigrosin staining $(55.63 \pm 6.64 \% ; P=0.654)$ and both indices were in correlation $(\mathrm{r}=0.504, P<0.05)$.

\section{Discussion}

For the prediction of semen quality, from an aspect of functionality, the assessment of the integrity of the sperm plasma membrane is of major significance because only spermatozoa with good function of the plasma membrane, allowing water movement in and out of the cell, can survive the preservation processes (Eilts 2005). The sperm plasma membrane is one of the key structures affected by cryopreservation. Thus the evaluation of the sperm plasma membrane is of utmost importance when establishing freezing protocols (Peña et al. 2005). The numerous functions of the membrane are related to the cell metabolism for maintaining sperm motility, capacitation, acrosome reaction, interactions between the spermatozoa and the epithelium of the female genital tract, and sperm-egg interactions (Rodriguez-Martinez 2003). If sperm membrane integrity is disturbed, the cell loses its ability to maintain homeostasis, which leads to an influx of water and extracellular ions.

A great deal of attention has been focused on the role of simple HOS test in connection with sperm membrane integrity evaluation. Currently, this method is widely used in both assisted reproduction in human medicine (World Health Organization 2010) and in farm animal semen evaluation (Corea and Zavos 1994).

Jeyendran et al. (1984) first introduced HOS test as an indicator that assesses the functional integrity of the human sperm membrane and described its relationship to other semen characteristics. Vazquez et al. (1997) suggested that hypoosmotic swelling test (HOST) is a sensitive and reproducible test to assess the functional integrity of boar sperm membranes and may be a useful tool for detecting subpopulations of subviable spermatozoa when used in conjunction with another type of membrane integrity test. The original HOS test subjected the semen sample to $30 \mathrm{~min}$ of incubation in a hypoosmotic medium but as Pérez-Liano et al. (2001) found, the same results could be achieved with a short 5 min HOST. Nowadays, both time intervals are recommended as a standard for carrying out HOS test in human semen samples (World Health Organization 2010). Unlike other sperm indicators, short HOST result presented a significant correlation with in vivo fertility in boars and indicated possible damage due to cold shock (Pérez-Liano et al. 


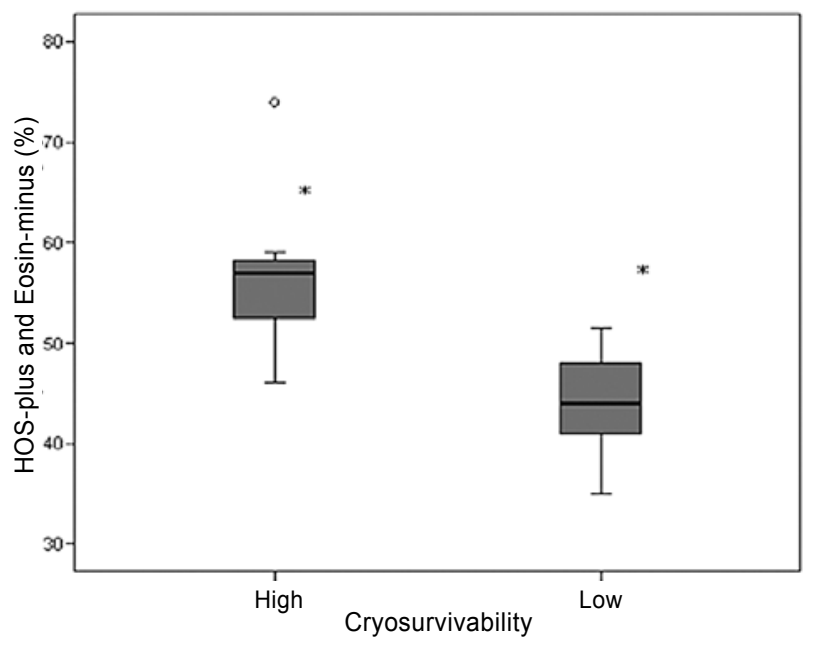

Figure 2. Percentage of HOS-positive and eosin-negative spermatozoa in the group of boar semen samples with high and low cryosurvivability. $* P<0.01$.

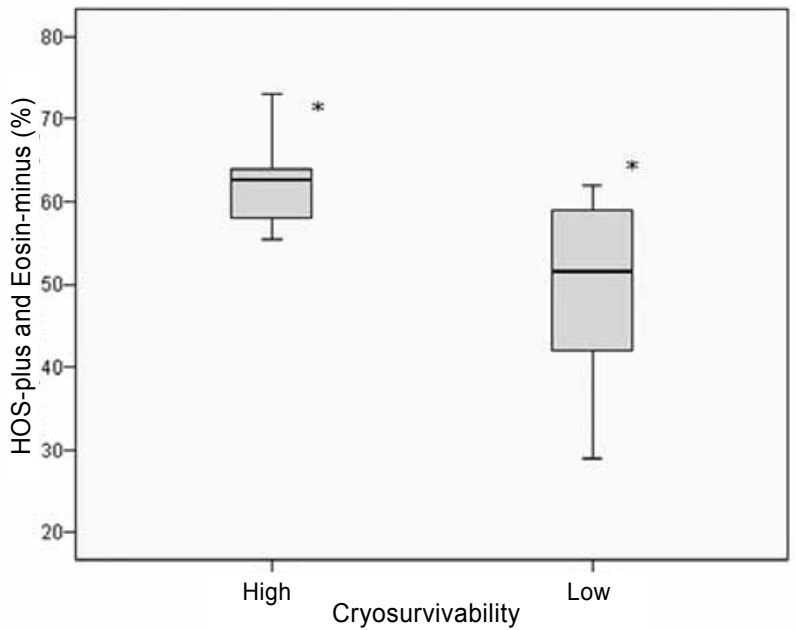

Figure 3. Percentage of HOS-positive and eosin-negative spermatozoa in the group of bull semen samples with high and low cryosurvivability. $* P<0.01$.

2001). According to the authors' conclusion, the short HOST data significantly improved the effectiveness of routine semen analysis tests in predicting fertility. On the other hand, Rota et al. (1999) discovered that short HOS test has its limitations when testing in vitro fertility of bovine spermatozoa since the test did not appear to be sufficiently sensitive to discriminate between semen samples of intermediate fertility like those used in the study.

Combined supravital staining with hypoosmotic sperm swelling test has already been used by Chan et al. (1991). Their results in human males gave evidence that the test shows a good correlation to the sperm penetration assay, which in turn is predictive of 
successful clinical in vitro fertilization. Also Tartaglione and Ritta (2004) reported that the combination of HOST and supravital test with eosin staining had a greater potential to predict fertility of bull semen than each test used separately.

In a previous study (Věžník et al. 2010) we confirmed that modified HOS test is a suitable method for showing functional changes in sperm membrane integrity by comparing results of mHOST and sperm survival rate after a 2 -h incubation of semen in saline $(2 \mathrm{~h}$ sperm survival test).

In the present study, we tried to ascertain whether mHOST could be a suitable method for the prediction of individual differences in the semen of both boars and bulls. A higher percentage of sperm was recovered after the thawing of ejaculates with a higher percentage of HOS-positive and eosin-negative sperm, whereas no other indicator of the routine sperm analysis testing used in this study could predict the sensitivity of spermatozoa to the cryopreservation process. Mean values for fresh sperm motility in bulls were found even slightly higher $(P<0.05)$ in the group determined as bad freezers.

It is to the advantage of mHOST that it is easy to perform and the laboratory equipment required is undemanding in comparison to the other hitherto published methods evaluating the membrane functional integrity in relation to sperm freezability focused e.g. on the detection of early membrane changes, such as Annexin V or YOPRO-1 (Sion et al. 2004; Peña et al. 2007), which require a fluorescence microscope or flow cytometer.

In conclusion, based on the obtained results, simple HOS test in combination with intravital staining (modified HOS test) seems to be a procedure providing additional information concerning dynamic changes in the sperm membrane resistance. Even though the presented results were so far done on a limited number of animals, we can assume that the test can detect differences in sperm membrane resistance which allows assessment of semen quality from the perspective of its further use, e.g. cryopreservation.

\section{Acknowledgements}

The authors would like to thank laboratory technician Marie Svobodová for her assistance in semen analyses and Mr. Paul Veater (Bristol, United Kingdom) for proofreading the translated manuscript. Funded by QI111A166 and CEITEC - Central European Institute of Technology (ED1.1.00/02.0068) from the European Regional Development Fund.

\section{References}

Amann RP 1989: Can the fertility potential of a seminal sample be predicted accurately? J Androl 10: 89-98

Casas I, Sancho S, Briz M, Pinart E, Bussalleu E, Yeste M, Bonet S 2010: Fertility after post-cervical artificial insemination with cryopreserved sperm from boar ejaculates of good and poor freezability. Anim Reprod Sci 118: $69-76$

Chan PJ, Tredway DR, Corselli J, Pang SC, Su BC 1991: Combined supravital staining and hypoosmotic swelling test. Hum Reprod 6: 1115-1118

Correa JR, Zavos PM 1994: The hypoosmotic swelling test: its employment as an assay to evaluate the functional integrity of the frozen-thawed bovine sperm membrane. Theriogenology 42: 351-360

Eilts BE 2005: Theoretical aspects of canine semen cryopreservation. Theriogenology 64: 692-697

Hammerstedt RH 1979: Characterization of sperm surface using physical techniques. In: Fawcett DW and Bedford JM (Eds). The spermatozoon: Maturation, Motility, Surface Properties and Comparative Aspects. Urban and Schwarzenberg, Baltimore, USA, pp. 205-216

Holt WV, Medrano A, Thurston LM, Watson PF 2005: The significance of cooling rates and animal variability for boar sperm cryopreservation: insights from the cryomicroscope. Theriogenology 63: 370-382

Jeyendran RS, Van der Ven HH, Perez-Pelaez M, Crabo BG, Zaneveld LJD 1984: Development of an assay the functional integrity of the human sperm membrane and its relationship to other semen characteristics. J Reprod Fert 70: 219-228

Kruger TF, Menkveld R, Stander FSH, Lombard CJ, Van der Merwe JP, Van Zyl JA, Smith K 1986: Sperm morphologic features as a prognostic factor in vitro fertilization outcome. Fertil Steril 46: 1118-1123

Parks JE, Lynch DV 1992: Lipid composition and thermotropic phase behavior of boar, bull, stallion, and rooster sperm membranes. Cryobiology 29: 255-266 
Peña FJ, Saravia F, Johannisson A, Walgren M, Rodríguez-Martínez H 2005: A new and simple method to evaluate early membrane changes in frozen-thawed boar spermatozoa. Int J Androl 28: 107-114

Peña FJ, Saravia F, Johannisson A, Wallgren M, Rodríguez-Martínez H 2007: Detection of early changes in sperm membrane integrity pre-freezing can estimate post-thaw quality of boar spermatozoa. Anim Reprod Sci 97: 74-83

Pérez-Liano B, Lorenzo JL, Yenes P, Trejo A, Garcia-Casado P 2001: A short hypoosmotic swelling test for the prediction of boar sperm fertility. Theriogenology 56: $387-98$

Roca J, Hernández M, Carvajal G, Vázquez JM, Martinez EA 2006: Factors influencing boar sperm cryosurvival. J Anima Sci 84: 2692-2699

Rodriguez-Martinez H 2003: Laboratory semen assessment and prediction of fertility: still utopia? Reprod Domest Anim 38: 312-318

Rota A, Penzo N, Vincenti L, Mantovani R 1999: Hypoosmotic swelling (HOS) as a screening assay for testing in vitro fertility of bovine spermatozoa. Theriogenology 53: 1415-1420

Sion B, Janny L, Boucher D, Grizard G 2004: Annexin V binding to plasma membrane predicts the quality of human cryopreserved spermatozoa. Int J Androl 27: 108-114

Tartaglione CM, Ritta MN 2004: Prognostic value of spermatological parameters as predictors of in vitro fertility of frozen-thawed bull semen. Theriogenology 62: 1245-1252

Thurston LM, Watson PF, Holt WV 1999: Sources of variation in boar spermatozoa fertility following cryopreservation. Cryobiology 39: 355

Vazquez JM, Martinez EA, Martinez P, Garcia-Artiga C, Roca J 1997: Hypoosmotic swelling of boar spermatozoa compared to other methods for analysing the sperm membrane. Theriogenology 4: 913-922

Veznik Z, Matouskova O, Svecova D, Zajicova A 2001: The use of the computer technology for the evaluation of the strict morphological sperm analysis. Vet Med-Czech 46: 35-40

Věžník Z, Přinosilová P, Zajícová A, Kunetková M, Hlavicová J 2010: Evaluation of sperm membrane resistance in boars by modification of HOS test (in Czech).Veterinářství 60: 695-697

Westendorf P, Richter L, Treu H 1975: Deep freezing of boar sperma. Laboratory and insemination results using the Hülsenberger paillet method (in German). Dtsch Tierarztl Wochenschr 82: 261-267

World Health Organization 2010: WHO Laboratory Manual for the Examination of Human Semen. 5th ed. WHO Press, Geneva, Switzerland, 271 p.s 
Plate V

Přinosilová P. et al.: Modified hypoosmotic ... pp. 313-319
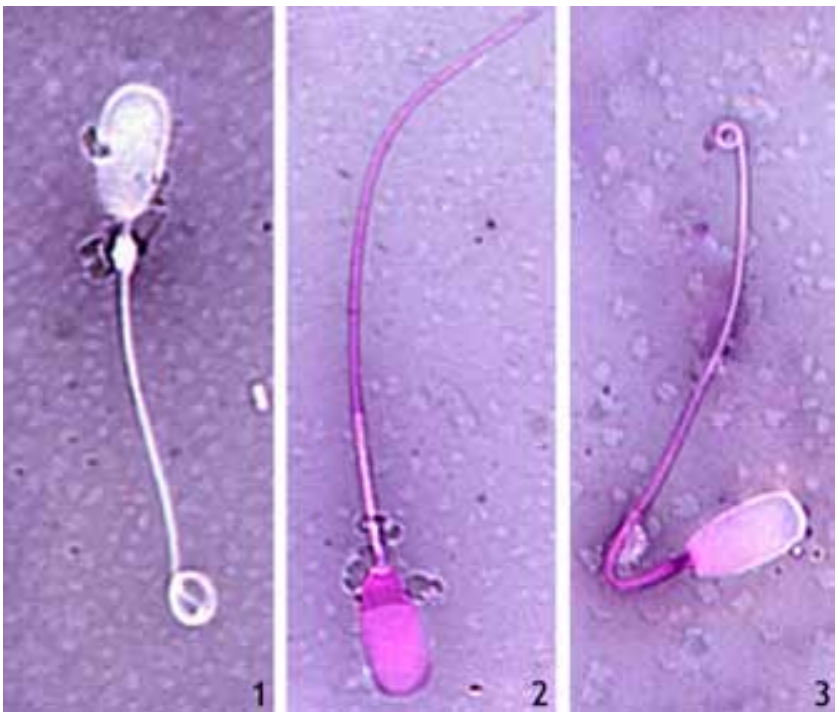

Fig. 1. 1) HOS-positive and eosin-negative, i.e. live spermatozoa with good membrane resistance, 2) HOSnegative and eosin-positive, i.e. dead spermatozoa and 3) HOS-positive and eosin-positive, i.e. viable spermatozoa but with reduced plasma membrane resistance. 